\title{
Optique de Bragg-Fresnel multicouche pour le rayonnement X-UV
}

\author{
J.-M. André et R. Barchewitz \\ Laboratoire de Chimie Physique-Matière et Rayonnement, Université Pierre et Marie Curie, \\ UA 176 du CNRS, 11 rue Pierre et Marie Curie, 75231 Paris cedex 05, France
}

\begin{abstract}
Résumé: Le principe des lentilles de Bragg-Fresnel multicouches est donné. Les techniques de fabrication sont résumées. Les performances de ces optiques sont discutées. Des exemples d'application et les perspectives concernant leur développement sont présentés.
\end{abstract}

\begin{abstract}
The principle of Bragg-Fresnel multilayer lenses is given. The techniques of manufacturing are summarized. The performances of these optics are discussed. Examples of application and perspectives concerning their development are presented.
\end{abstract}

\section{Introduction}

Une des fonctions principales de l'optique est de focaliser la lumière en particulier pour réaliser des images ou concentrer l'énergie lumineuse. Pour le rayonnement visible cette fonction est réalisée dans la grande majorité des cas par des lentilles transparentes fonctionnant sur le principe de la réfraction. Dans le domaine du rayonnement $X$, de telles lentilles ne sont pratiquement pas utilisables car le phénomène de réfraction est peu important, ce qui signifie plus précisement qu'un rayon est très peu dévié à la traversée d'un dioptre. Cette situation est due au fait que les indices optiques relatifs dans le domaine du rayonnement $X$ sont toujours très proches de l'unité et qu'en conséquence de la seconde loi de Descartes, l'angle de réfraction reste très voisin de l'angle d'incidence. Par ailleurs dans le domaine du rayonnement $X$ la matière reste toujours notablement absorbante ce qui interdit la fabrication de lentilles "épaisses".

Pour réaliser une focalisation dans ce domaine spectral, on a donc recours soit à des systèmes à miroirs soit à des lentilles de Fresnel. Les systèmes à miroirs fonctionnent:

- soit dans le régime de la quasi-réflexion totale sous incidence rasante, par exemple dans les systèmes de type Wolter

- soit dans le régime de réflexion sélective de Bragg obtenu à l'aide d'un film 
multicouche sous incidence proche de la normale, par exemple dans les systèmes de type Schwarzschild.

Ces dispositifs à miroirs qui présentent l'avantage d'offrir une grande ouverture sont malheurement difficiles à construire et à utiliser. Aussi leurs préfère-t-on dans un certain nombre de cas, en particulier pour la construction de microscopes $X$, les lentilles de Fresnel dont le principe sera rappelé ultérieurement. Les travaux de Baez [1] ont amorcé l'utilisation de ces lentilles pour le rayonnement de courte longueur d'onde. Les lentilles de Fresnel destinées au rayonnement $\mathrm{X}$ et fonctionnant par transmission sont actuellement très employées en microscopie X [2]. Récemment V.V. Aristov et ses collaborateurs ont proposé, puis largement développé, un nouveau type de lentille de Fresnel fonctionnant non pas par transmission, mais par réflexion dans les conditions sélectives de Bragg [3]. Cette réflexion de Bragg est assurée soit par des cristaux naturels, soit par des miroirs interférentiels muiticouches. $\mathrm{Ce}$ sont ces derniers systèmes qui font l'objet de cet article. Notons enfin que plus récemment encore, il a été développé des lentilles de Fresnel fonctionnant dans le régime de réflexion quasi totale sous incidence très rasante, dans le but d'élargir la bande passante[4].

\section{Principe des lentilles de Fresnel}

Considérons une source lumineuse monochromatique de longueur d'onde $\lambda$ située au point $B$ et un point $A$ quelconque de l'espace situé à une distance $2 \mathrm{~L}$ de $B$ (voir Fig.1). L'ensemble des points $E$ tels que le chemin optique (BEA) corresponde à une phase donnée est un ellipsoïde de révolution de foyers situés en $\mathrm{A}$ et $\mathrm{B}$, dit ellipsoïde isophase.

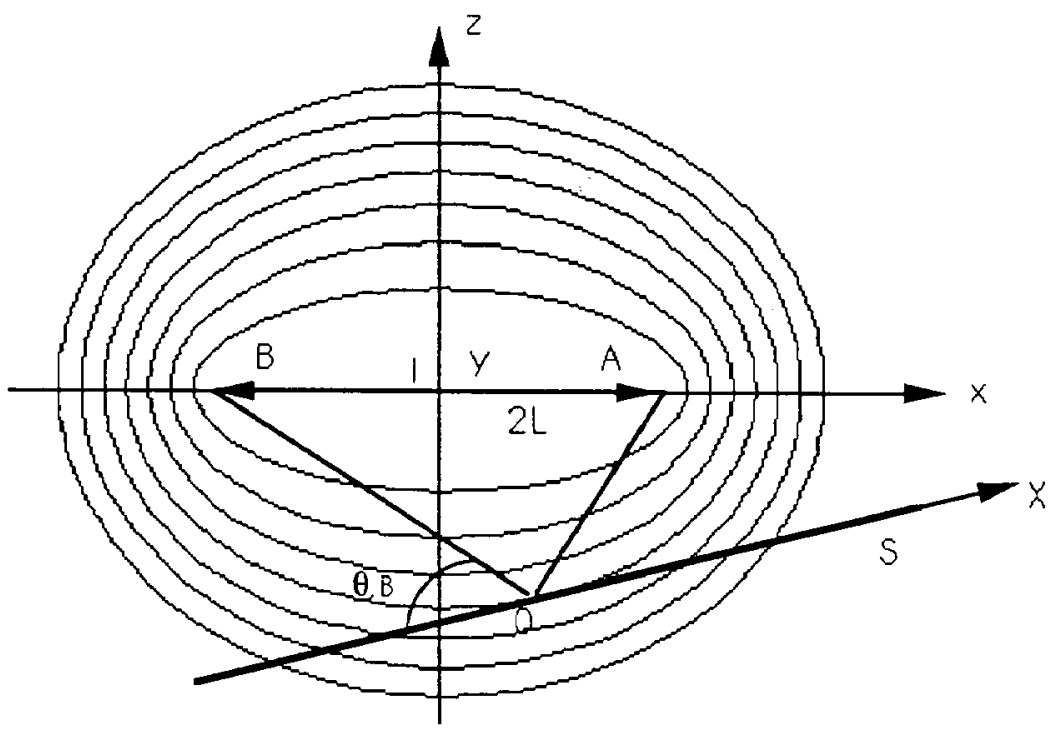

Fig.1: Géométrie permettant de décrire le principe des lentilles de Fresnel. Les ellipses représentent la projection de quelques ellipsoïdes équiphases.

Les ellipsoïdes isophases qui définissent des chemins optiques différant d'un multiple de $\lambda / 2$ ont pour équation cartésienne dans le repère $(I, x, y, z)$ 


$$
\frac{x^{2}}{a^{2}}+\frac{y^{2}}{b^{2}}+\frac{z^{2}}{b^{2}}=1_{(1 a),}^{a}=\frac{L}{2}+n \frac{\lambda}{2}(1 b), b^{2}=a^{2}-\left(\frac{L}{2}\right)^{2}
$$

Tous les points $\mathbf{L}$ compris entre deux ellipsoïdes consécutifs forment un lieu géométrique $\Lambda$ caractérisé par le fait que le terme de phase associée au chemin optique (BLA) possède le même signe. Si l'on veut que le point A devienne l'image du point $B$ au moyen d'une optique située sur une surface $S$, il faut considérer l'intersection des lieux géométriques $\Lambda$ et de la surface $S$. L'ensemble de ces intersections définissent les zones de Fresnel. Pour obtenir l'image, il convient d'éliminer la contribution (régime du "réseau d'amplitude") ou d'inverser le signe du terme de phase (régime du "réseau de phase") d'une zone sur deux. En effet les ondes correspondant à une zone de Fresnel sur deux interfèrent constructivement au point A puisque les termes de phase associés sont de même signe. En général la surface $S$ sera un simple plan de sorte que les limites des zones de Fresnel seront des ellipses dont les équations dans le repère cartésien $(0, X, y)$ indiqué sur la figure 1, s'écrivent:

$$
\frac{\mathrm{X}^{2}}{\mathrm{~K}^{2}}+\frac{\mathrm{y}^{2}}{\mathrm{~K}^{2}\left(\mathrm{~A}^{2}-\mathrm{L}^{2}\right)}=1
$$

avec

$$
\begin{aligned}
& X=x+\frac{U}{V}(2 b), \quad A=L+m \frac{\lambda}{4}, \text { m entier }(2 c), K=\sqrt{1+\frac{1}{U}+\left(\frac{U}{V}\right)^{2}-\frac{W}{U}}(2 d) \\
& U=\frac{\cos ^{2} \theta}{A^{2}}+\frac{\sin ^{2} \theta}{A^{2}-L^{2}}(2 e), \quad V=\frac{x_{O} \cos \theta}{A^{2}}+\frac{z_{O} \sin \theta}{A^{2}-L^{2}}(2 f), \quad W=\frac{x_{O}^{2}}{A^{2}}+\frac{z_{O}^{2}}{A^{2}-L^{2}}
\end{aligned}
$$

Le point $\mathrm{O}$ a pour coordonnées $\left(\mathrm{x}_{\mathrm{O}}, 0, \mathrm{z}_{\mathrm{O}}\right)$ dans le repère $(\mathrm{I}, \mathrm{x}, \mathrm{y}, \mathrm{z})$ et l'angle $\theta$ est lié à l'angle $\theta_{B}$ et aux distances $d_{2}=O A$ et $d_{1}=O B$, par la relation

$$
\theta=\operatorname{Arctg}\left[\left|\frac{d_{1}-d_{2}}{d_{1}+d_{2}}\right| \operatorname{tg} \theta_{B}\right]
$$

Les équations (2) définissent un réseau d'ellipses non confocales dont le rapport du petit axe au grand axe est voisin du sinus de l'angle $\theta$.

Dans le cas particulier usuel de lentilles fonctionnant par transmission ou par réflexion sous incidence quasi-normale, le plan $S$ est situé perpendiculairement à l'axe Ix. Les ellipses définissant les frontières des zones de Fresnel deviennent alors des cercles dont les rayons $\mathrm{R}_{\mathrm{m}}$ sont approximativement donnés à partir des équations (2),(3) en négligeant les termes quadratiques en $\lambda$, par

$$
\mathrm{R}_{\mathrm{m}} \approx \sqrt{\mathrm{mf} \lambda}
$$

où $f=d_{1} d_{2} /\left(d_{1}+d_{2}\right)$ est la distance focale de la lentille mince correspondant à la lentille de Fresnel.

Pour les lentilles fonctionnant par réflexion sous incidence oblique, il est aussi possible d'introduire la notion de distance focale $f$ et de grandissement $G=$ $\mathrm{d}_{2} / \mathrm{d}_{1}$, et de réécrire les équations (2) des ellipses sous une forme "simplifiée" 
négligeant les termes quadratiques en $\lambda$ :

$$
\left(x+m \frac{1-G}{1+G} \frac{\cos \theta}{\sin ^{2} \theta}\right)^{2}+\frac{y^{2}}{\sin ^{2} \theta}=m \frac{2 f \lambda}{\sin ^{2} \theta}
$$

Notons que dans le cas d'une focalisation à une dimension (un point source donne comme image un segment linéaire ), les frontières des zones de Fresnel dégénèrent en segments de droite dont la distance au centre de la lentille est donnée par la relation

$$
\mathbf{R}_{\mathrm{m}}^{ \pm}=\frac{\mathrm{n} \lambda}{\mathrm{m} \operatorname{tg} \theta \sin \theta}\left[\sqrt{1+\operatorname{tg}^{2} \theta\left(1+\frac{4 \mathrm{f}}{\mathrm{m} \lambda}\right)} \pm 1\right]
$$

On notera l'asymétrie de la structure des lentilles linéaires. Par analogie avec la diffraction par des réseaux, on parlera de fonctionnement en configuration standard lorque les traits sont perpendiculaires au vecteur d'onde incident et en configuration conique lorsque les traits sont parallèles au vecteur d'onde incident.

Dans le cas des lentilles de Bragg-Fresnel, le plan $S$ est partiellement occupé par un réflecteur de Bragg travaillant au voisinage de l'angle $\theta_{\mathrm{B}}$. Rappelons que ce réflecteur de Bragg est un dispositif interférentiel constitué par la répétition périodique d'un motif. Si on note $d$ la période correspondante, alors la longueur d'onde $\lambda$, l'angle d'attaque $\theta_{\mathrm{B}}$ et la période $d$ doivent pratiquement vérifier la condition d'interférence constructive, connue sous le nom de loi de Bragg, qui s'écrit

$$
2 \mathrm{~d} \sin \theta_{\mathrm{B}}=\mathrm{p} \lambda, \mathrm{p} \text { entier }
$$

Nous verrons par la suite que cette loi doit être légèrement modifiée pour tenir compte des effets de réfraction. On pratiquera dans ce réflecteur, suivant les ellipses définissant les frontières des zones de Fresnel, une gravure donnant lieu à une structure en relief. On obtient ainsi une succession de traits, à profil rectangulaire, constitués de multicouche et de sillons vides. Nous donnerons dans la section suivante des explications sur les techniques de gravure. Si la lentille fonctionne en "réseau d'amplitude", la gravure sera destinée à éliminer physiquement une zone de Fresnel sur deux; dans ce cas la gravure au niveau des sillons se fera jusqu'au substrat. On parlera par analogie avec la photographie de lentille positive ou négative (voir figure 2). Si la lentille fonctionne en "réseau de phase", la gravure aura pour but de décaler perpendiculairement à la surface, les zones de Fresnel adjacentes, d'une hauteur H égale à :

$$
\mathrm{H}=\frac{\varphi \lambda \sin \theta_{\mathrm{B}}}{2 \pi}
$$

où $\varphi$ désigne le déphasage associé à la différence de marche introduite par la hauteur $\mathrm{H}$ du relief. Suivant le principe du réseau de phase, la valeur du déphasage doit être égale à un multiple impair de $\pi$. En fait il faut souligner que, en particulier lorsque le réflecteur est un miroir interférentiel, la lentille "de phase" est rarement purement de ce type et qu'une modulation d'amplitude est généralement présente. 


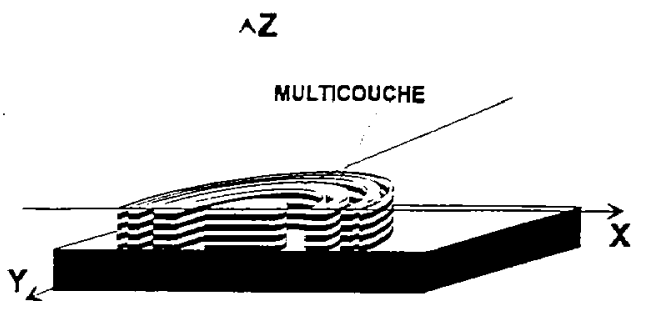

Fig.2: Représentation schématique d'une lentille de Fresnel d'amplitude positive. Dans le cas d'une lentille négative, les positions des traits et sillons sont inversées.

\section{Fabrication des lentilles de Bragg-Fresnel multicouches}

La fabrication d'une lentille est une opération qui s'effectue en plusieurs étapes résumées dans la figure 3 et qui fait appel aux techniques de dépôt de films minces à l'échelle nanométrique, à la lithographie par faisceau d'électrons et aux techniques de gravure ionique réactive (Reactive Ionic Etching R.I.E.) dans un plasma fluoré. Des compromis doivent être trouvés dans le choix des matériaux. Ceux-ci doivent offrir la possibilité de réaliser un miroir multicouche à forte réflectivité à la longueur d'onde souhaitée ce qui nécessite des matériaux présentant un constraste d'indice optique suffisamment élevé et donnant des interfaces lisses sans trop d'interdiffusion. Un grand nombre d'études a été consacré à ce problème. Il faut de plus que ces matériaux soient susceptibles de subir une attaque R.I.E assez rapide en donnant des motifs de bonne qualité, en particulier en terme de profil.

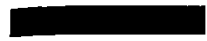

1) Substrat de SIlicium poli

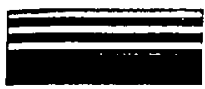

2) Dipôt d'une mulțcousche

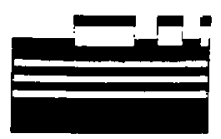

5) Depot de Nickel (30 nm)

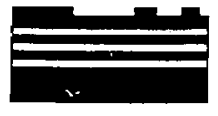

6) "Lith-or" du Nickel. El!mination do la risine et du mital

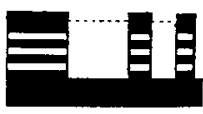

7) Gravure lonique ractive

dani un plasma fluord

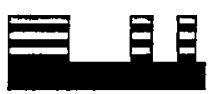

8)

4) Ecrtture par falsceau d'blectrons

et developpement de In ressine PMMA

Fig 3: Méthode de fabrication d'une lentille de Bragg-Fresnel multicouche. 


\section{Performances des lentilles de Bragg-Fresnel multicouches}

Les performances d'une lentille se discute principalement en termes d'efficacité, c'est à dire de taux de lumière focalisée dans un foyer, de résolution spatiale, c'est à dire d'aptitude à séparer spatialement les images de deux points sources rapprochés, voire de résolution spectrale. Ces résolutions conditionneront la fidélité de l'image à l'objet, problème très important en imagerie. Une approche fructueuse pour aborder globalement le problème des performances consiste à calculer l'amplitude de diffusion d'une lentille en champ moyen, c'est à dire dans le cadre de la diffraction de Fresnel, par un calcul d'électromagnétisme "rigoureux" tels que ceux qui ont été développés pour les réseaux de diffraction. Ce type de calcul a été effectué dans le cas d'une lentille linéaire éclairée par une source à l'infini [5] et une comparaison avec des résultats expérimentaux a été réalisée [8]. La figure 4 montre comparativement la répartition de l'intensité lumineuse diffractée par une lentille d'amplitude linéaire positive, mesurée (Fig4.a) et calculée (Fig4.b).
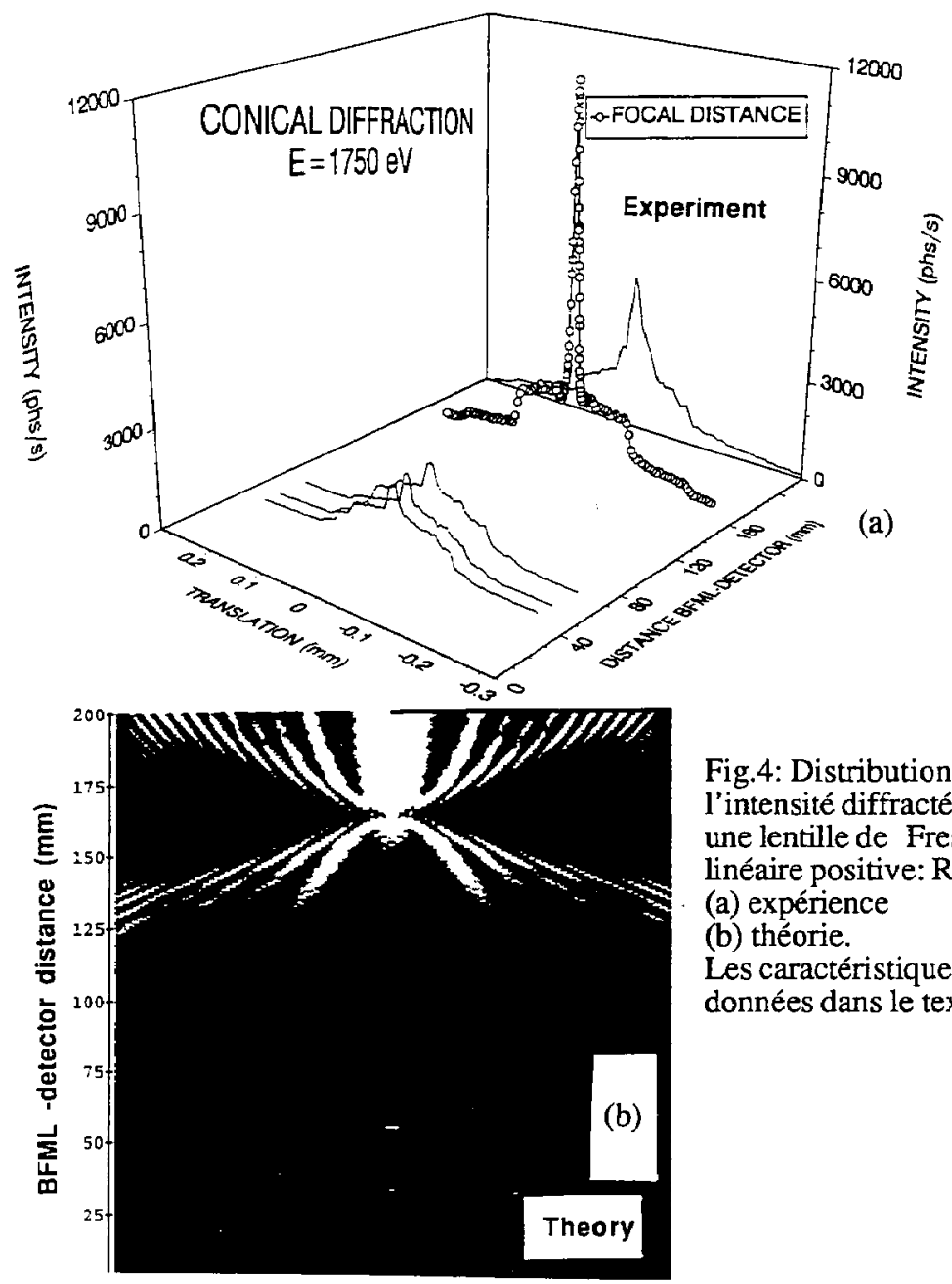

Fig.4: Distribution de l'intensité diffractée par une lentille de Fresnel linéaire positive: Ref[8]. (a) expérience (b) théorie.

Les caractéristiques sont données dans le texte. 
La lentille a pour dimension $\left(210 * 5000 \mu \mathrm{m}^{2}\right)$, la largeur de là zone centrale et de la zone externe est de 11 et 0,4 microns respectivement. La multicouche comporte 25 périodes de W/Si égales à $5,3 \mathrm{~nm}$. L'étude expérimentale a été réalisée à la longueur d'onde de $0,7 \mathrm{~nm}(1750 \mathrm{eV})$ à l'aide du rayonnement synchrotron délivré par Super-ACO (LURE à Orsay), en configuration conique, la source d'un diamètre voisin de $1 \mathrm{~mm}$ étant située à environ $15 \mathrm{~m}$ de la lentille. La distance focale de la lentille (qui est indépendante de sa nature positive ou négative mais dépend de la configuration standard ou conique) est égale à 164 mm pour la longueur d'onde considérée. L'accord entre l'expérience et le théorie est remarquable, en particulier, en ce qui concerne la distribution de l'intensité en dehors des foyers; ainsi la théorie prévoit la présence de lumière derrière le foyer d'ordre 1 pour la lentille positive et la situation opposée pour la lentille négative, faits qui ont été confirmés expérimentalement. De tels calculs peuvent être étendus à des lentilles elliptiques éclairées par des sources situées à distances finies, mais ils sont particulièrement "gourmands" en temps de calcul. Aussi est-il intéressant de dégager quelques règles et critères de performances.

\section{1 efficacité:}

On distinguera le cas des lentilles d'amplitude et des lentilles de phase.

- lentille d'amplitude: La théorie électromagnétique de la diffraction de Fresnel appliquée aux zones de Fresnel d'amplitude [5] permet de montrer que l'efficacité au foyer d'ordre $m=2 j+1$ avec $j$ entier, s'écrit sous la forme

$$
\varepsilon_{\mathrm{m}}^{\mathrm{ampl}}=\frac{1}{\pi^{2}} \frac{\bar{\varepsilon}}{\mathrm{m}^{2}}
$$

où $\bar{\varepsilon}$ représente la réflectivité de la structure de Bragg gravée. Celle-ci s'obtient par un calcul standard de réflectivité pic de miroir multicouche [7] en introduisant à la place des indices optiques réels des matériaux des indices optiques moyennés que l'on pourrait qualifiés d'effectifs, pour tenir compte de la présence de volumes vides introduits par la présence des sillons. La modification de constante optique du milieu par suite de la gravure affecte non seulement la valeur de l'efficacité pic mais aussi la valeur de l'angle de Bragg optimal et la largeur de la bande passante angulaire par rapport au miroir multicouche non gravé. La valeur de l'angle de Bragg ainsi que la largeur de la bande passante pour la lentille sont diminuées par rapport au miroir (non gravé) [5].

- lentille de phase: La situation est un peu plus compliquée que dans le cas précédent mais on peut écrire approximativement l'efficacité au foyer d'ordre $\mathrm{m}=2 \mathrm{j}+1$ comme suit

$$
\varepsilon_{\mathrm{m}}^{\text {phase }}=\frac{2}{\pi^{2}} \frac{\bar{\varepsilon}}{\mathrm{m}^{2}}(1-\cos \varphi)
$$

où $\bar{\varepsilon}$ représente ici la contribution moyenne en terme d'efficacité, de la structure multicouche. L'utilisation de lentille de phase doit permettre une augmentation d'efficacité par rapport à la lentille d'amplitude dans un rapport voisin de 4.

\section{2 résolution spatiale et imagerie:}

La résolution spatiale est généralement estimée à partir du critère de Rayleigh, c'est à dire de la valeur du rayon $\delta$ de la première frange sombre de la figure d'Airy à laquelle on peut assimiler en première approche la figure de diffraction 
d'une lentille de Fresnel éclairée par une source à l'infini:

$$
\delta=1,22 \frac{\mathrm{IN}_{\mathrm{N}}}{\mathrm{m}}
$$

où $r_{N}$ est la largeur de la zone la plus exteme. La relation (11) montre que la résolution "point à point" est améliorée par la diminution de $\mathbf{r}_{\mathrm{N}}$ et par l'augmentation de l'ordre $\mathrm{m}$ (mais dans ce cas au détriment de l'efficacité selon les équations 9 et 10 ).

L'imagerie d'objets en particulier d'objets étendus est affectée par de nombreux problèmes. On notera principalement

- la résolution spatiale

- la présence d'un fond continu provenant essentiellement de l'ordre zéro; l'utilisation de lentilles de phase ou d'amplitude négatives plutôt que positives [5], l'obstruction de la zone centrale ou l'emploi de la lentille dans une configuration hors axe [8] peuvent être envisagés pour pallier ce problème.

- la limitation de la bande passante qui peut réduire le champ

- les phénomènes d'aberration, en particulier celui lié au fait que la structure réflectrice ne soit pas infiniment mince et que la gravure ne reproduit pas dans l'épaisseur de celle-ci la forme elliptique des frontières de zones [9].

\section{Applications et perspectives}

Les lentilles de Bragg-Fresnel devraient rapidement trouver des applications pratiques dans les problèmes de microfocalisation et d'imagerie. En ce qui concerne la microfocalisation, on vise la réalisation de microsondes donnant une tache focale de dimension inférieure à $10 * 10 \mu \mathrm{m}^{2}$. Une microsonde à fluorescence fonctionnant dans le domaine $\mathrm{X}$ dur ( 8 à $12 \mathrm{keV}$ ) et utilisant une lentille de phase offrant une résolution submicronique a été réalisée et décrite récemment dans la littérature [10]. Dans le domaine $X$ mou, ces microsondes pourraient être surtout intéressantes pour la spectromicroscopie d'électrons.

Les applications en imagerie sont actuellement peu développées mais devraient connaitre un essor prochain en particulier pour le diagnostic des sources plasmas et le contrôle des sources synchrotrons. Des premiers résultats avec des cristaux comme réflecteurs de Bragg ont été rapportés dans la littérature [11].

L'intérêt des lentilles de Bragg-Fresnel comparées aux lentilles fonctionnant par transmission réside dans leur résistance mécanique et thermique, et dans la possibilité de les utiliser dans le domaine du rayonnement $X$ dur où il est difficile de trouver des matériaux suffisamment absorbants pour les lentilles fonctionnant par transmission. Un des inconvénients majeurs des lentilles de Fresnel demeure leur faible ouverture; cependant les progrès technologogiques permettent de prévoir l'agrandissement des tailles utiles jusqu'à des dimensions correspondant au cône central des onduleurs installés sur les sources synchrotrons de troisième génération [11].

[1] Baez A. V., J. Opt. Soc. Am. 51(1961) 405-412

[2] X-ray microscopy III (Michette A.G., Morrison G. R., Buckley C. J. Eds, Springer-Verlag, Berlin, 1992)

[3] Aristov V. V. et al., Rev. Phys. Appliquée (1988)1623-1630; Erko A. I., J. X-Ray Sci. Techno. 2(1990)297-316.

[4] Yu. A.Basov et aI., Opt. Comm. 109(1994)324-327

[5] Sammar A., André J.-M., J. Opt. Soc. Am. 10(1993) 2334-2337.

[6] Idir M. et al., Proc. 4-th Conference on X-ray Microscopy (Russia, 1993)

[7] Pardo B.et al, Rev. Phys. App. (1988)1579-1597

[8] Idir M. et al., Proc. 4-th Conference on X-ray Microscopy (Russia, 1993)

[9] Dhez P. et al., Appl. Opt.31(1992)6662-6667.

[10] Erko A. I. et al., Opt. Comm. 106(1994),146-149.

[11] Tarazona E. et al., Rev. Sci. Instrum. 65(1994)1959-1963. 